\title{
Analysis of LO phonon properties in III-nitrides: interaction with carriers and microscopic analysis
}

Ishitani, Y., Oki, K., Chizaki, M., Okamoto, S., Nakayama, T., et al.

Y. Ishitani, K. Oki, M. Chizaki, S. Okamoto, T. Nakayama, B. Lin, B. Ma, K. Morita, H. Miyake, D. lida, K. Ohkawa, "Analysis of LO phonon properties in III-nitrides: interaction with carriers and microscopic analysis," Proc. SPIE 11686, Gallium Nitride Materials and Devices XVI, 116861Y (5 March 2021); doi: $10.1117 / 12.2576776$

SPIE. Event: SPIE OPTO, 2021, Online Only 


\title{
Analysis of LO phonon properties in III-nitrides: interaction with carriers and microscopic analysis
}

\author{
Y. Ishitani*a, K. Oki a , M. Chizaki ${ }^{\text {a }}$, S. Okamoto ${ }^{\text {a }}$, T. Nakayama ${ }^{\text {a }}$, B. Lin ${ }^{\text {a }}$, B. Ma ${ }^{\text {a }}$, K. Morita ${ }^{\text {a }}$, H. \\ Miyake $^{\mathrm{b}}$, D. Iida ${ }^{\mathrm{c}}$, and K. Ohkawa ${ }^{\mathrm{c}}$ \\ ${ }^{a}$ Graduate School of Electric and Electronic Engineering, 1-33, Yayoicho, Inage-ku, Chiba, 263- \\ 8522, Japan \\ ${ }^{\mathrm{b}}$ Graduate School of Electrical and Electronic Engineering, Mie University \\ 1577 Kurimamachiya, Tsu, 514-8507, Japan \\ ${ }^{\mathrm{c}}$ Computer, Electrical and Mathematical Sciences and Engineering Division, King Abdullah \\ University of Science and Technology, Thuwal, 23955-6900, Saudi Arabia
}

\begin{abstract}
Longitudinal optical (LO) phonon has strong electric interaction with particles and fields. Particularly, the interaction in III-nitrides is more significant than that in conventional III-V materials. We show phonon-exciton interaction properties in experimental PL spectrum analysis and theoretical calculation of population transfers of excitonic levels. Thermally nonequilibrium occupations of LO phonons and other modes generated by the LO-phonon decomposition are thought to shift the population distribution in principal quantum number states and kinetic energy to the higher energy side. The radiative exciton recombination lifetime is determined by the population distribution in the excitonic states, which is determined by the balance of the electronic and phononic elementary processes. The interaction of excitons and phonons releases the excess energy to the thermal bath of the lattice system, which sometimes yields negligible lattice temperature increase in the excited region or the nonequilibrium state between electron and phonon systems. A Raman scattering imaging measure is introduced to exhibit spatial transport of phonons generated by the energy relaxation and nonradiative recombination of the excited electrons and holes, where pump-probe measurements are enabled by the simultaneous irradiation of two laser beams. It is found that the phonon transport is blocked by the misfit dislocations located on a $\mathrm{Ga}_{0.84} \mathrm{In}_{0.16} \mathrm{~N} / \mathrm{GaN}$ heterointerface.
\end{abstract}

Keywords: LO phonon, phonon-exciton interaction, PXR model, phonon transport, microscopic Raman imaging

\section{INTRODUCTION}

III-nitride materials have strong electron-phonon interaction. It is widely known that the electrons are highly scattered by longitudinal optical (LO) phonons. Exciton dynamics shows various properties depending on temperature and electronphonon interaction. Although free-exciton photoluminescence is observed even at room temperature (RT) ${ }^{[1]}$ laser emission is mostly dominated by electron-hole plasma. It has been reported that exciton-polariton lasing at RT takes place at a carrier density of $4.5 \times 10^{16} \mathrm{~cm}^{-3}$, whereas it is disturbed at the density of $2 \times 10^{17} \mathrm{~cm}^{-3}$, which is still under the Mott transition density. ${ }^{[2]}$ It is possible that LO phonons formed by the energy relaxation of electrons excite excitonic states, and dissociate excitons. When a GaN sample is excited by a photon with a wavelength of $266 \mathrm{~nm}$, for example,11 LO phonons are estimated to be emitted by the energy relaxation of electrons and holes. However, we have little information on electronhole-exciton dynamics and energy transport in this excited spatial region when considering the processes with phonons or whole energy species. The increase in temperature of excitons in this thermal nonequilibrium field has been reported in literatures ${ }^{[3,4]}$, however the dynamics by integrating the lattice and electron systems have not been elucidated. The exciton dynamics is investigated in many pieces of literature using rate equations by considering only the electronic levels, while we show, in this discussion, the requirement of the analysis, including all the energy species.

Gallium Nitride Materials and Devices XVI, edited by Hiroshi Fujioka, Hadis Morkoç, Ulrich T. Schwarz Proc. of SPIE Vol. 11686, 116861Y · ( 2021 SPIE · CCC code: 0277-786X/21/\$21 doi: $10.1117 / 12.2576776$ 
We have been studying carrier and exciton dynamics by taking into account phonons, which are energy carriers in the excited region: phononic-excitonic-radiative (PXR) model, where phonon absorption and emission processes have been introduced as well as the exciton-electron (hole) collision processes ${ }^{[5,6]}$ Here, we have presented exciton dynamics analysis based on a set of rate equations by taking into account phononic processes of longitudinal acoustic (LA) phonon and LO phonon. Toward our simulation model's practical application, we extend the discussion to the system with donors in the present analysis. The radiative recombination lifetime of exciton, which is a crucial factor for light-emitting devices, is discussed for bulk materials of GaN. Previously, various dependence of this lifetime on temperature has been discussed in many literatures ${ }^{[7-9]}$, where the law of $3 / 2$ power of temperature is valid in some limited temperature range. This value is theoretically based on the condition that the population is localized in the $1 S$ state. We discuss this power value by using our PXR model in universal cases, and the discussion is extended to the thermodynamic viewpoint.

When we intend to control the electron-phonon interaction dynamics, spatial phonon transport analysis is thought to be required. We have reported studies on Raman scattering using simultaneous irradiation of two laser beams: one for heating and the other for Raman signal probing. ${ }^{[10]}$ We show the phonon-transport analysis of in-plane and across the interface using GaInN/GaN samples.

\section{METHOD}

\subsection{Experimental}

Experimental analysis of excitonic radiation properties was conducted using a $6.0 \mu \mathrm{m}$-thick GaN film grown on a substrate of selective-area-growth $\mathrm{GaN}$ with thickness of $9.7 \mu \mathrm{m}$ on a sapphire wafer grown by metal-organic vapor phase epitaxy (MOVPE) method. ${ }^{[1]}$ The respective values of full-width at half maximum values of (0004) and (10-11) X-ray rocking curves were 290 and 383 (arcsec). The electron density measured by infrared reflectance (IRR) analysis at RT was less than $5 \times 10^{16} \mathrm{~cm}^{-3} . \mathrm{Ga}_{x} \mathrm{In}_{1-x} \mathrm{~N}$ samples with a thickness of $110 \mathrm{~nm}$ were grown on $\mathrm{n}-\mathrm{GaN}$ layers on sapphire substrates by a MOVPE system. ${ }^{[12]}$ The alloy composition $x$ values were in the range of $0.84-0.95$. The layer of $\mathrm{Ga}_{0.84} \mathrm{In}_{0.16} \mathrm{~N}$ is found to be partially relaxed by x-ray diffraction analysis. No geometrical surface structure was found for these samples in the images observed by optical microscope or scanning electron microscope. An IRR analysis revealed the residual electron density of this film was approximately $6 \times 10^{17} \mathrm{~cm}^{-3}$.

In the PL measurements of the GaN film, the $267 \mathrm{~nm}$ line of the third harmonic wave of a Ti: $\alpha-\mathrm{Al}_{2} \mathrm{O}_{3}$ laser with a pulse duration of $150 \mathrm{fs}$ was utilized. The excitation laser power of $1 \mathrm{~mW}$ for the $267 \mathrm{~nm}$ line was estimated to generate electron-hole pairs of $1.4 \times 10^{15} \mathrm{~cm}^{-3}$ per pulse. The PL measurement system consisted of a monochromator $(F=25 \mathrm{~cm})$ and a streak camera. In the Raman measurements of $\mathrm{Ga}_{x} \mathrm{In}_{1-x} \mathrm{~N}$ and the underlaying GaN layers, a 325-nm laser was used for photoexcitation of carriers in the $\mathrm{Ga}_{x} \mathrm{In}_{1-x} \mathrm{~N}$ layer and the resultant heating and also for Raman measurements of the excited layer. A 532-nm laser was used for the Raman measurement of the GaN layer. The 532-nm light was focused with a diameter of approximately $0.65 \mu \mathrm{m}$, while the $325-\mathrm{nm}$ light was diffused at an approximate diameter of $4 \mu \mathrm{m}$. The fluctuation of circumstance temperature was kept less than 1 degree. The Raman shift was calibrated using the $5460.7 \AA$ line of a Hg lamp. The density of free carriers generated by a $1 \mathrm{~mW}$ excitation of the $325-\mathrm{nm}$ beam was estimated to be approximately $5.55 \times 10^{13} \mathrm{~cm}^{-2}$ or less by considering the lifetime shorter than 500 ps. Carrier diffusion in the $\mathrm{Ga}_{x} \mathrm{In}_{1-x} \mathrm{~N}$ layers further decreases the carrier density to approximately one quarter. In simultaneous excitation, the carrier density is estimated to be less than $10^{17} \mathrm{~cm}^{-3}$ for the excitation laser power of $10 \mathrm{~mW}$ for $x=0.83$. In these cases, the thermal energy conduction by free carriers can be neglected ${ }^{[10]}$.

\subsection{Phononic-excitonic- radiative model}

Numerical simulation of the exciton dynamics was conducted using the following rate equations, where rate or rate coefficients of the respective elementary processes were calculated using theoretical formulae. ${ }^{[5,6]}$ The processes taken into account were Fröhlich interaction of the LO phonons, deformation and piezoelectric interactions of the LA phonon, electron-collisional processes, and radiative recombination processes. The states with principal quantum number $n=1$ to 5 of A exciton were taken into account as a simple model. The binding energy of $E_{B}$ of GaN was taken as $27 \mathrm{meV}$. The activation energy of donor $E_{\mathrm{D}}$ was taken as $15 \mathrm{meV}$. The rate or rate coefficients of the electron-collisional processes are referred to the formulae for hydrogen plasma. ${ }^{[13]}$ The dispersion relations of the LO phonon and LA phonon were taken into account. Population densities of each exciton state $N_{\mathrm{X}}(n)$, free electron $N_{\mathrm{e}}$, and free hole were numerically obtained by solving the following set of rate equations. 


$$
\begin{aligned}
& \frac{d}{d t} N_{\mathrm{x}}(n)=\sum_{n^{\prime} \neq n}\left[A\left(n^{\prime}, n\right)+C\left(n^{\prime}, n\right) N_{\mathrm{e}}+W_{\mathrm{XX}}\left(n^{\prime}, n\right)\right] N_{\mathrm{X}}\left(n^{\prime}\right)+\left[\beta(n)+\alpha(n) N_{\mathrm{e}}+W_{\mathrm{CX}}(n)\right] N_{\mathrm{e}}^{2} \\
& \quad-\left\{\sum_{n^{\prime} \neq n}\left[A\left(n, n^{\prime}\right)+C\left(n, n^{\prime}\right) N_{\mathrm{e}}+W_{\mathrm{XX}}\left(n, n^{\prime}\right)\right]+S(n) N_{\mathrm{e}}+W_{\mathrm{XC}}(n)+P_{\mathrm{rec}}(n)\right\} N_{\mathrm{X}}(n), \\
& \frac{\mathrm{d}}{\mathrm{d} t} N_{\mathrm{h}}=\sum_{n}\left[S(n) N_{\mathrm{e}}+W_{\mathrm{XC}}(n)\right] N_{\mathrm{X}}(n)+R_{\mathrm{VC}}-\sum_{n}\left[\beta(n)+\alpha(n) N_{\mathrm{e}}+W_{\mathrm{CX}}(n)+B\right] N_{\mathrm{e}} N_{\mathrm{h} .}
\end{aligned}
$$

The densities of total, neutral, and ionized donors are represented by $N_{\mathrm{D}}, N_{\mathrm{D}}{ }^{0}$ and $N_{\mathrm{D}}{ }^{+}$, respectively. For these densities, the relation of $N_{\mathrm{D}}=N_{\mathrm{D}}{ }^{+}+N_{\mathrm{D}}{ }^{0}$ is valid. The relation between the densities of these states are expressed by the following Shana-Boltzmann's relation.

$$
\frac{N_{\mathrm{e}} N_{\mathrm{D}}^{+}}{N_{\mathrm{D}}^{0}}=\left(\frac{2 \pi m_{\mathrm{e}} k_{\mathrm{b}} T}{h^{2}}\right)^{3 / 2} \exp \left(-\frac{E_{\mathrm{D}}}{k_{\mathrm{b}} T}\right) .
$$

$k_{\mathrm{b}}$ is the Boltzmann factor. $A\left(n, n^{\prime}\right), C\left(n, n^{\prime}\right)$ and $W_{\mathrm{Xx}}\left(n, n^{\prime}\right)$ are the rates or rate coefficients of transitions from the $n$ state to $n^{\prime}$ state by radiative, electron-collisional, and phononic processes, respectively. $P_{\mathrm{rec}}(n)$ is the radiative recombination rate of the state $n . \beta(n), \alpha(n)$, and $W_{\mathrm{CX}}(n)$ are the exciton formation rate coefficients of radiative, electron-collisional (threebody), and phononic processes, respectively. $S(n)$ is the exciton dissociation rate coefficient by the electron-collisional process. $W_{\mathrm{xc}}(n)$ is the rate of exciton dissociation by phononic processes. $\mathrm{B}$ is the radiative recombination rate coefficient of free electrons and holes. Temperatures of electron $T_{\mathrm{e}}$, exction $T_{\mathrm{X}}$, lattice $T_{\mathrm{L}}$, and the rate of electron excitation from the valence band to conduction band $R_{\mathrm{VC}}$ were taken as parameters. Here, the direct formation of each $n$ state of exciton $R_{\mathrm{Vx}}(n)$ from the vacuum level was excluded. The kinetic energy distributions of electrons and excitons were obtained by the Maxwell-Boltzmann distribution function. The phonon occupation factor $n_{\mathrm{q}}$ was obtained by the Bose-Einstein distribution function.

\section{RESULTS AND DISCUSSIONS}

\subsection{Exciton-phonon interaction in GaN}

Figure 1(a) shows the temporal development of PL spectrum of the $\mathrm{u}-\mathrm{GaN}$ when the excitation laser power is $1 \mathrm{~mW}$. The spectra were analyzed by the following formulae of the constituent transition processes. The observed spectrum line width of $2.6 \mathrm{meV}$ for the $n=1$ state of free A exciton FA(1) is greater than the critical width $1.5 \mathrm{meV}$ of exciton-photon coupling. ${ }^{[14]}$ The exciton scattering lifetime estimated from the line width is approximately $1.2 \mathrm{ps}$ or shorter, which indicates that the polaritons are scattered at a high rate in the order of $10^{11} \mathrm{~s}^{-1}$. Thus, the zero phonon lines (ZPLs) of excitons were fitted by quasi-Voigt function. The spectra of two-electron transition (TET) and its LO phonon replicas (LORs) were also fitted using this function. The function of $\left(E-E_{n}+m E_{\mathrm{LO}}\right)^{5 / 2-m} \exp \left[-\left(E-E_{n}+m E_{\mathrm{LO}}\right) / k_{\mathrm{b}} T\right]$ was used for one phonon-related LOR (1LOR, $m=1)$ and 2LORs $(m=2)$ of free excitons. Here, $E_{n}$ is the energy of the $n$ state of free
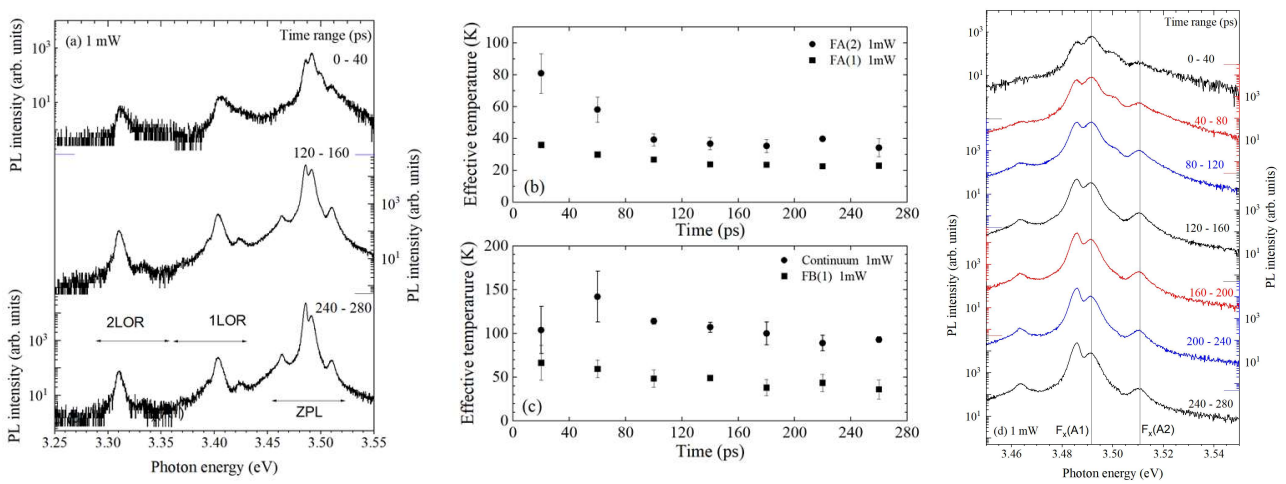

Figure 1 Characteristic properties of excitonic luminescence, (a): temporal development of the PL spectrum in the energy range of ZPL, 1LOR, and 2LOR, (b) and (c): temporal developments of $T_{\mathrm{X}} \mathrm{S}$ of $\mathrm{FA}(n), \mathrm{FB}(1)$, and $T_{\mathrm{e}}$, for the excitation power of $1 \mathrm{~mW}$ and (d): magnified spectra in the ZPL region. 
exciton. The ZPL spectrum of the continuum was fitted by a function proportional to $\left(E-E_{\mathrm{g}}\right)^{1 / 2} \exp \left[-\left(E-E_{\mathrm{g}}\right) / k_{\mathrm{b}} T\right]$. $(E-$ $\left.E_{\mathrm{g}}+m E_{\mathrm{LO}}\right)^{3-m} \exp \left[-\left(E-E_{\mathrm{g}}+m E_{\mathrm{LO}}\right) / k_{\mathrm{b}} T\right]$ was adopted for its LOR spectra $(m=1$ for $1 \mathrm{LOR}$, and $m=2$ for $2 \mathrm{LOR}){ }^{[15,16]}$ The spectrum functions obtained by this process satisfactorily fitted the experimental data. ${ }^{[17]}$ As we have constraints for the energy regions and spectrum shapes of the constituent lines, the uniqueness of the fitting was obtained. Figures 1(b) and (c) shows the obtained free exciton temperatures of $\mathrm{FA}(1), n=2$ of the A exciton $\mathrm{FA}(2)$, and $n=1$ of the $\mathrm{B}$ exciton $\mathrm{FB}(1)$. This figure shows that the kinetic energy of exciton is under a thermally nonequilibrium state, which is thought to be caused by the absorption of phonons generated by the energy relaxation of photo-excited carriers, which has been confirmed by the spectra obtained for two excitation wavelengths of $337 \mathrm{~nm}$ and $266 \mathrm{~nm}$, where the generated carrier densities were adjusted to be the same. The difference is the carrier relaxation energy or the number of generated phonons. ${ }^{[4]}$

Figure 1(d) shows the magnified spectra in the region of ZPL. Although the exciton heating by the laser irradiation is observed, the peak energies of ZPLs are constant and independent of time. This result indicates that $T_{\mathrm{L}}$ is not increased even in the incipient time region after the laser-pulse irradiation when $T_{\mathrm{X}}$ and $T_{\mathrm{e}}$ are higher. It is considered that the electron system and phonon system are not in thermally equilibrium relationship. The expected mechanism is that kinetic energy of electrons and excitons is finally transformed to LA phonons, which covers wide spatial region or diffuses into large space, with the result that the temperature increase is negligible in the excited region.

\subsection{Analysis by Phononic-Excitonic-Radiative model}

Figure 2 shows the excitation and deexcitation population flows when $T_{\mathrm{e}}, T_{\mathrm{X}}$, and $T_{\mathrm{L}}$ agree and disagree. The calculation is conducted on the total electron density of $10^{16} \mathrm{~cm}^{-3}$ and $N_{\mathrm{D}}=0$ under the continuous excitation condition. When the system is at extremely low temperature, downward population transfer dominates for the states of $n=2$ and 3 , while, for the upper states, the transfer between the states $n$ and $n \pm 1$ or direct population exchange with the continuum states dominates. When any of the temperatures increases, the excitation flow from the $n=2$ state to the $n=3$ state or continuum states becomes significant, which makes the strong population coupling between the $n=2$ state and the continuum states. Particularly, the increase in exciton and phonon temperatures yield the direct population coupling between the $n=2$ state and the continuum states. This property agrees with the feature observed in the experimental result. ${ }^{[4,17]}$ At $300 \mathrm{~K}$, phononic processes dominates population flows among these states, and further, the direct population exchange between the continuum and each level dominates, which means the population distribution to excited $n$ states and extension of the radiative lifetime.

We exhibit a practically important example of the application of this model, that is, the radiative lifetime of exciton. This radiation is dominated by the recombination of electrons and holes via FA(1). It is reported that the radiative exciton lifetime is proportional to $T^{3 / 2}$ in bulk crystals, ${ }^{[5]}$ which is based on the relation of $\hbar^{2} K^{2} / 2 M=3 k_{\mathrm{b}} T / 2$ and the small recombination window at $K \sim 0$. Here, $K$ and $M$ are the wavevector and mass of exciton. This relation of $T^{3 / 2}$ is valid when we assume that excitons occupy only the state of $\mathrm{FA}(1)$, whereas it is not a universal rule when we take into account other exciton states with different energy levels: FA(2), continuum states, and so forth. We have calculated the population lifetime of the FA(1) excitons by the instantaneous excitation of electrons at the density of $1 \times 10^{15} \mathrm{~cm}^{-3}$. The decay rate of the population was obtained by the exponential fitting of the calculated temporal development of the population density. In the temperature range lower than $50 \mathrm{~K}$, the constant lifetime due to exciton-polariton is assumed. The population lifetime of FA(1) increases to more than $100 \mathrm{~ns}$ at $300 \mathrm{~K}$ and the law of $T^{3 / 2}$ is not valid when we assume the maximum $n\left(n_{\max }\right)$ of 5 as shown in the undoped case of Fig. 3. This result agrees with the experimental radiative lifetime reported by $\operatorname{Im}$ et al, where the radiative lifetime has been obtained by decomposing a PL decay rate to radiative and nonradiative components

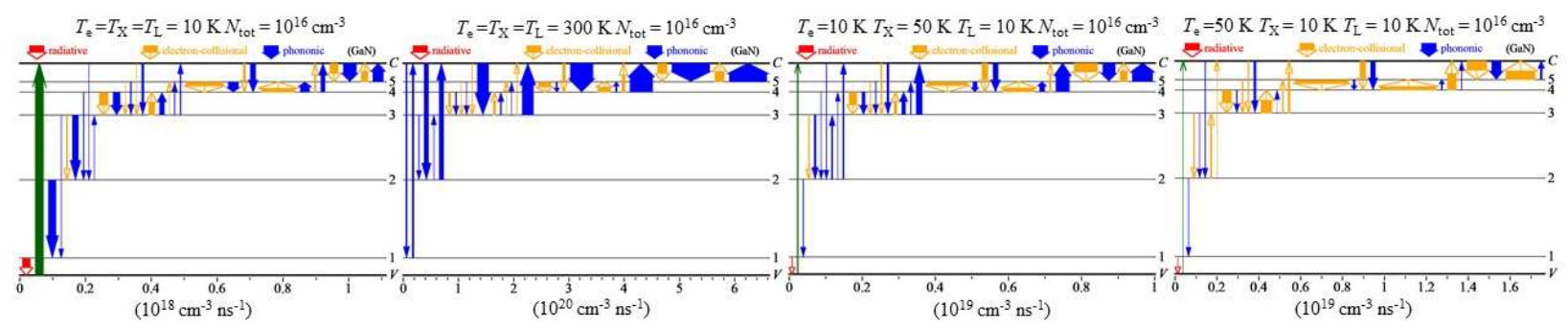

Figure 2 Population transfer flows for thermally equilibrium and nonequilibrium cases. The rulers indicated below the respective figures give the calibration of the thickness of arrows or population transfer rates. 
using the dependence of the PL decay rate and intensity on temperature. ${ }^{[7]}$ This result indicates that the populations in higher $n$ states work as reservoirs of the FA(1) population. However, in another experimental result, the law of $T^{3 / 2}$ is valid to approximately $100-200 \mathrm{~K} \cdot{ }^{[9]}$ Some references exhibit longer lifetime values in the higher temperature range. ${ }^{[7,8]}$. When we take into account the background electrons and the broadening of energy states, which reduce the effective binding energy of excitons and $n_{\max }$. The dependence of the population lifetime of $\tau_{\mathrm{d}}$ for FA(1) on temperature for the donor density $N_{\mathrm{D}}$ of $4 \times 10^{17} \mathrm{~cm}^{-3}$ and $n_{\max }=1$, is plotted in Fig. 3. It is found that the dependence of $\tau_{\mathrm{d}}$ approaches to the line of $T^{3 / 2}$. Here, it is thought that the key factors reducing the increase in the lifetime are that the reduction of the number of influential sublevels or $n_{\max }$ and the population imbalance between electrons and holes. The process of [electron + hole $\rightarrow$ exciton] indicates that the increase in electron density enhances the association to exciton, which simultaneously suggests the decrease in the energy of the exciton system. In this case, it is estimated that the energy is released to the thermal bath via
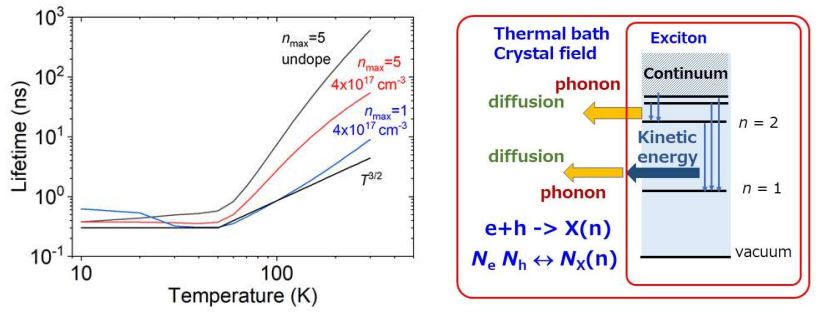

Figure 3 Dependence of radiative lifetime of FA(1) population on temperature. The right-hand side figure shows the model of thermal relaxation.

phonons. Thus, the analysis of spatial energy transport including phonon is required in the discussion of the carrier dynamics. In the following section, the methodology to detect and analyze thermal energy transport is discussed.

\subsection{Analysis of phonon transport through $\mathrm{GaInN/GaN}$ heterointerfaces}

The geometric condition of the present Raman imaging is depicted in Fig. 4(a). The wavenumber shift of the $\mathrm{E}_{2}$ (high) mode $\left(\mathrm{E}_{2}{ }^{\mathrm{H}}\right)$ peak of the $\mathrm{GaN}$ under layer, $\Delta \mathrm{E}_{2}{ }^{\mathrm{H}}(\mathrm{GaN})$, by irradiating the $325-\mathrm{nm}$ pump (heating) laser with $20 \mathrm{~mW}$ is plotted in Fig. 4(b) for the sample of $\mathrm{Ga}_{0.95} \mathrm{In}_{0.05} \mathrm{~N} / \mathrm{GaN} / \alpha-\mathrm{Al}_{2} \mathrm{O}_{3}$, where the probing spot was scanned from the heating spot. The $\mathrm{E}_{2}^{\mathrm{H}}$ peak wavenumber shift to the lower side is attributed to the increase in the temperature. The increase in temperature is mitigated as the probing position is away from the heating spot. It is thought that the phonon transported is detected to approximately $20 \mu \mathrm{m}$ in the lateral direction from the $325-\mathrm{nm}$ laser spot. An example of PL intensity mapping for the $\mathrm{Ga}_{0.84} \mathrm{In}_{0.16} \mathrm{~N} / \mathrm{GaN} / \alpha-\mathrm{Al}_{2} \mathrm{O}_{3}$ sample is plotted in Fig. 4(c), where a clear triangle structure is observed. It is thought that the dark region has high misfit dislocations (MDs). $\Delta \mathrm{E}_{2}{ }^{\mathrm{H}}(\mathrm{GaN})$ at the same region as shown in Fig. 4(c) is plotted in Fig. 4(d). ${ }^{[10]}$ This figure exhibits the same triangular structure. The higher temperature increase is observed inside the triangular structure with lower MDs. This result indicates that the phonon transport is blocked at the MDs. Further, the wraparound phonon transport via the higher quality region is negligible. The wavenumber shift observed for this sample is higher than that observed for the sample of $\mathrm{Ga}_{0.95} \mathrm{In}_{0.05} \mathrm{~N}$, where, it is thought, the phonon conduction is higher than the sample of $\mathrm{Ga}_{0.84} \mathrm{In}_{0.17} \mathrm{~N}$. The analysis of the sample of $\mathrm{Ga}_{0.84} \mathrm{In}_{0.16} \mathrm{~N}$ indicates that the phonon transport at the heterointerface is quasi ballistic.

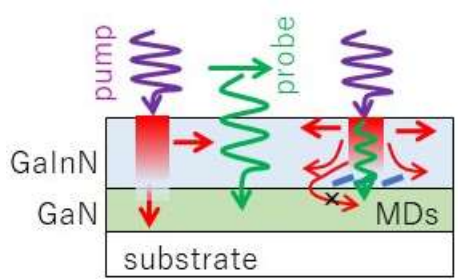

(a)

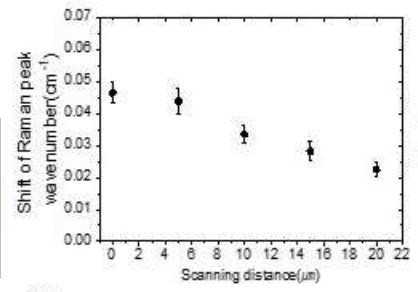

(b)

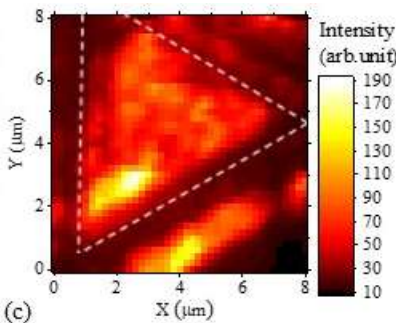

(c)

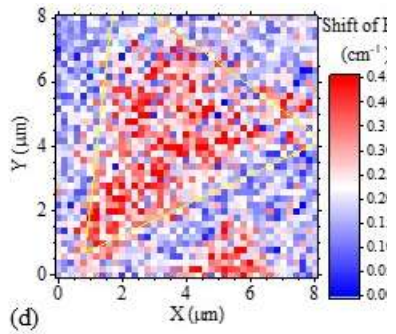

(d)

Figure 4 Energy shift of the $\mathrm{E}_{2}{ }^{\mathrm{H}}$ of the $\mathrm{GaN}$ layer by the simultaneous irradiation of the 532-nm and 325-nm lasers on $\mathrm{Ga}_{x} \mathrm{In}_{1-x} \mathrm{~N} / \mathrm{GaN}$ heterointerface, (a) geometric condition of measurement, (b) $\Delta E_{2}{ }^{\mathrm{H}}$ by scanning the probe-laser spot for $x=0.95$, (c) mapping image of PL intensity, (d) $\Delta \mathrm{E}_{2}{ }^{\mathrm{H}}$ for $x=0.84$. 


\section{CONCLUSION}

In the PL analysis of $\mathrm{u}-\mathrm{GaN}$ at low temperature and the excitation density in the order of $10^{16} \mathrm{~cm}^{-3}$, we have detected the increase in electron and exciton temperature, whereas the increase in the lattice temperature is negligible. The PXR model shows that the radiative lifetime of exciton is determined by the population flows among the principal quantum number states. The imbalance of electron and hole densities and the broadening of excitonic states enhance the radiative recombination rate. The deexcitation to the 1S state is thought to be caused by the energy release to the thermal bath of the lattice system yielding negligible lattice temperature increase in the excited region. Phonon transports have been analyzed for samples of $\mathrm{Ga}_{x} \mathrm{In}_{1-\mathrm{x}} \mathrm{N} / \mathrm{GaN}$ heterostructures by pump-and-probe Raman scattering images using double lasers. We have detected the blocking of phonon transport to the GaN underlayer at MDs located on the GaInN/GaN heterointerface.

\section{ACKNOWLEDGEMENTS}

This study was partly supported by the Grant-in-Aid for Scientific Research of the Japan Society for the Promotion of Science (JP16H06415, JP16H06425, JP17H02772) and King Abdullah University of Science and Technology (KAUST) (BAS/1/1676-01-01)

\section{REFERENCES}

[1] S. Chichibu, T. Azuhata, T. Sota, and S. Nakamura, "Excitonic emissions from hexagonal GaN epitaxial layers", J. Appl. Phys. 79, 2784 (1996).

[2] P. Bhattacharya, T. Frost, S. Deshpande, M. Z. Baten, A. Hazari, and A. Das, "Room Temperature Electrically Injected Polariton Laser", Phys. Rev. Lett. 112, 236802 (2014).

[3] D. Hägele, R. Zimmermann, M. Oestreich, M. R. Hofmann, W. W. Rühle, B. K. Meyer, H. Amano, and I. Akasaki, "Cooling dynamics of excitons in GaN", Phys. Rev. B 59, R7797 (1999).

[4] Y. Ishitani, K. Oki, and H. Miyake, "Statistics of excitonic energy states based on phononic-excitonic-radiative model", Jpn. J. Appl. Phys. 58, SCCB34 (2019).

[5] K. Oki and Y. Ishitani, "Influence of LO and LA phonon processes on thermal-nonequilibrium excitation and deexcitation dynamics of excitons in GaN, AlN, and ZnO”, J. Appl. Phys. 125, 205705 (2019).

[6] K. Oki, B. Ma, and Y. Ishitani, "Population decay time and distribution of exciton states analyzed by rate equations based on theoretical phononic and electron-collisional rate coefficients", Phys. Rev. B 96, 205204 (2017).

[7] J. S. Im, A. Moritz, F. Steuber, V. Härle, F. Scholz, and A. Hangleiter, "Radiative carrier lifetime, momentum matrixelement, and hole effective mass in GaN", Appl. Phys. Lett. 70, 631 (1997).

[8] C. Hauswald, P. Corfdir, J.K. Zettler, V.M. Kaganer, K.K. Sabelfeld, S. Fernández-Garrido, T. Flissikowski, V. Consonni, T. Gotschke, H.T. Grahn, L. Geelhaar, and O. Brandt, "Origin of the nonradiative decay of bound excitons in GaN nanowires" Phys. Rev. B 90, 165304 (2014).

[9] S. F. Chichibu, A. Uedono , K. Kojima, H. Ikeda, K. Fujito, S. Takashima, M. Edo, K. Ueno, and S. Ishibashi, "The origins and properties of intrinsic nonradiative recombination centers in wide bandgap GaN and AlGaN", J. Appl. Phys. 123, 161413 (2018).

[10] S. Okamoto, N. Saito, K. Ito, B. Ma, K. Morita , D. Iida, K. Ohkawa, and Y. Ishitani, "Energy transport analysis in a $\mathrm{Ga}_{0.84} \mathrm{In}_{0.16} \mathrm{~N} / \mathrm{GaN}$ heterostructure using microscopic Raman images employing simultaneous coaxial irradiation of two lasers", Appl. Phys. Lett. 116, 142107 (2020).

[11] H. Miyake, N. Masuda, Y.Ogawahara, M. Narukawa, K. Hiramatsu, T. Ezaki, and N. Kuwano, "Growth of crackfree AlGaN on selective-area-growth GaN", J. Cryst. Growth 310, 4885 (2008).

[12] K. Ohkawa, F. Ichinohe, T. Watanabe, K. Nakamura, and D. Iida, "Metalorganic vapor-phase epitaxial growth simulation to realize high-quality and high-In-content InGaN alloys", J. Cryst. Growth 512, 69 (2019).

[13] T. Fujimoto, [Plasma Spectroscopy], Clarendon Press, Oxford, U.K., Chapter 43 (2004).

[14] B. Monemar, P. P. Paskov, J. P. Bergman, A. A. Toropov, T. V. Shubina, T. Malinauskas, and A. Usui, "Recombination of free and bound excitons in GaN", Phys. Stat. Sol. B 245, 1723 (2008).

[15] B. Segall and G. D. Mahan, "Phonon-Assisted Recombination of Free Excitons in Compound Semiconductors", Phys. Rev. 171, 935 (1968).

[16] S. J. Xu, G. Q. Li, S.-J. Xiong, and C. M. Che, "Temperature dependence of the LO phonon sidebands in free exciton emission of GaN", J. Appl. Phys 99, 073508 (2006).

[17] Y. Ishitani, K. Takeuchi, N. Oizumi, H. Sakamoto, B. Ma, K. Morita, H. Miyake, and K. Hiramatsu, "Excitation and de-excitation dynamics of excitons in a GaN film based on the analysis of radiation from high-order states", J. Phys. D 49, 245102 (2016). 